there were, above and a little to the left side of the pubes, two sinuses admitting a probe some distance inwards and downwards towards the bladder, but not leading to anything definitely. The sinuses discharged slightly, but no urine seemed to come from them. On examining the bladder with a sound, and on passing the finger into the vagina, a calculus was felt in the bladder, apparently of large size.

On July 24th, under chloroform, the urethra was first dilated with the blades of a pair of urethral forceps and with the finger. A large lithotrite was then placed on the stone, and an attempt made to crush it, but the screw failed to act owing to the blades being too far apart, on account of the size of the stone. The lithotrite was withdrawn, and on further examination with the finger in the bladder, the points of a hair-pin were felt penetrating the coats of the bladder on the left side. It was decided to open the bladder above the pubes. An incision, about two and a half inches long, was made in the median line from the pubes upwards, and the parts dissected through till the bladder was reached, all bleeding vessels being secured on the way. The bladder was then fixed with a hook and opened, a pair of forceps introduced, and the stone caught. The main part was withdrawn with the hair-pin running through it ; a large portion of the stone at one end crumbled down, and the fragments were removed with a scoop. The wound in the bladder was now closed with four or five interrupted catgut sutures, and one or two vessels in its coats were tied. The edges of the wound in the abdominal walls were brought together with catgut sutures, the sutures being passed through the entire thickness of the muscles and integuments, as in the operation for ovariotomy, and a drainage-tube inserted. The operation was done under the carbolic spray, and the wound dressed antiseptically.

July 26th.-Recovered well from the chloroform, and appears bright and cheerful; has had very little pain, no sickness, or bad symptoms of any kind. Temperature $99^{\circ} 5^{\circ}$. The urine has continued to run away by the urethra, and apparently has not been blood-stained. Wound dressed, as the dressing became urinous.

During the next nine days she continued without a bad symptom; the wound being dressed from time to time, under the spray, and the drainage tube gradually shortened.

On Angust 6th the wound, which had continued closed throughout, was found all but healed, a small sinus, which the piece of drainage tube still kept open, remaining below. The sinuses, which had never discharged since the operation, had very nearly closed up; she had almost regained the power of retaining the urine. Her temperature the last two nights had been $101 \cdot 2^{\circ}$, but in every other respect she seemed exceedingly well and bright.

14th.-The wound and sinuses quite healed; very little urine now escapes involuntarily. Temperature still continues above normal, $100^{\circ}$ to $101^{\circ}$; but otherwise she appears perfectly well.

20th.-She is apparently quite recovered, and has full control over her urine, which is acid and clear; she has been out of bed. Went out quite well, and has continued so since.

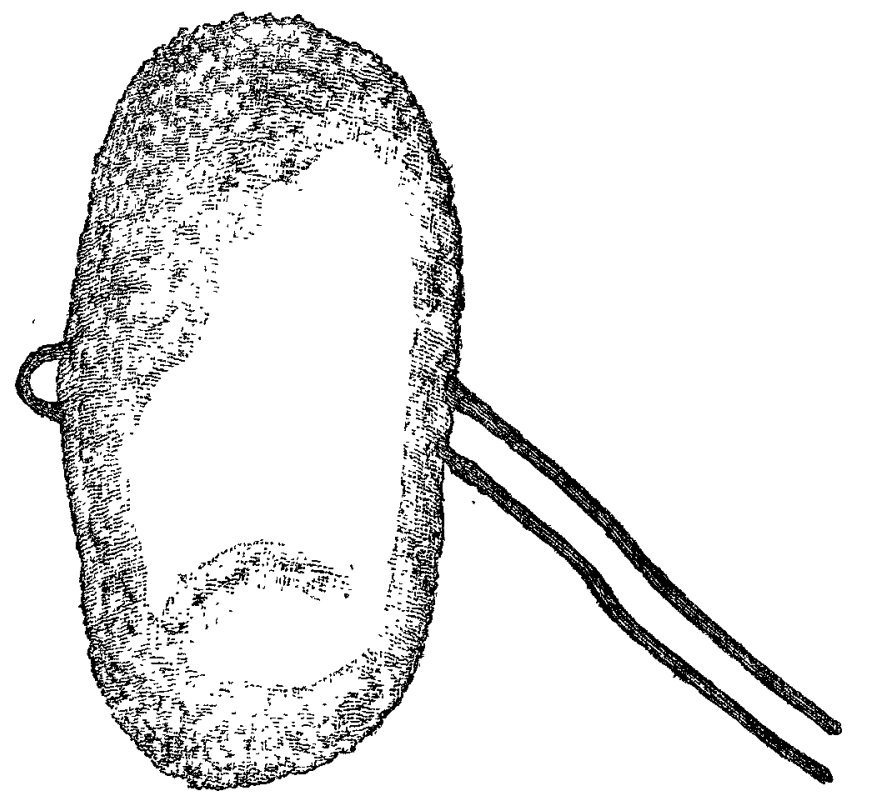

The woodcut represents the calculus in its entirety; the points of the hair-pin are shown in the position in which they were found in the bladder, penetrating its coats to about one inch and a half.

Devonport.

\section{ON SOME POINTS IN THE DIAGNOSIS OF PHTHISIS.}

BY A. B. SHEPHERD, M.D., F.R.C.P.,

PHYSICIAN TO THE HOSPITAL, YICTORIA PARIK; ASSISTANT-PHYSICIAIN TO ST. MARY'S HOSPITAL.

(Concluded from p. 11.)

I PASS over other collected cases, which, while they would take up too much time, illustrate only the same difficulty, and turn to the records of another master of diagnosis.

Addison ${ }^{1}$ has laid down that, "when any form of chronic induration of the pulmonary tissue exists, and especially if attended with dilated bronchial tubes, neither auscultation nor percussion enables us to distinguish snch a condition of lung from phthisical disease. If bronchitis be present, and the induration be situated at the apex, the signs are perfectly identical with those of phthisical disorganisation." And he adds, "Our diagnostic resources are to be found in the history and progress of the case, the absence of some of the more ordinary symptoms of phthisis, and some incongruity observable between the local signs and the general aspect and condition of the patient." Again, Dr. Walshe, who in 1848 allowed that cheesy infiltration of a portion of lung may be due to "a fatty transformation of catarrhal and pneumonic inflammation-products, as originally taught by Addison," tells us that " the real difficulty in diagnosis consists in the distinction between certain cases of chronic bronchitis and phthisis, ... especially when dilatation of the bronchi exists ;" and some pages further on gives certain marks of difference by which the distinction may be established; at the same time the distinctions asserted in no way contradict the sentence I have just quoted from Addison, representing, I think, most fairly the existing difficulty.

For the difficulty does exist for each one of us. I have made a brief abstract of the notes of a case now under my care in the Victoria Park Hospital, which shows how very nearly it is brought home to myself.

The patient is a labourer, forty-two years of age, admitted into hospital on the 5th of last November, married, of dark complexion, 5 feet 9 inches in height, and then weighing 9st. 5lb. On these last two points it may be remembered that Dr. Brinton in his little book on "Life Assurance" (p. 32), lays down that an adult male in good health, 5 feet 6 inches in stature, ought to weigh rather more than 10st. The patient tells us that since he first became ill, eighteen months ago, he has lost 2 st., which would bring him well above Dr. Brinton's law. The only disease he has had was ague, eighteen years ago. His father is eighty years old, with probably chronic arthritis ; his mother is eighty, and healthy. He has two brothers and a sister, all in good health. His present illness began with dry cough and pain in the left side; later the cough was accompanied by expectoration. After violent retching, three months ago, he brought up, he thinks, four ounces of clotted blood, and has occasionally seen streaks since; he has had no night sweats for the last six months, though he had had previously. On admission his pulse is 96 ; his temperature $98.2^{\circ} \mathrm{Fahr}$.; his general functions normal; his finger-nails not curved. There is marked dulness and tubular breathing at the right apex, to fourth rib; intense bronchitis over both lungs. Further notes by my clerk, Mr. Macdonald, to whom I am indebted for what I have read, are these : cavernous respiration and crepitation at right base in front; harsh breathing and small crepitations in supra-spinous fossa, extending to base. On the left side resonance on percussion; harsh breathing and crepitation cleared by cough. All the bronchitic sounds increased ; there was from time to time abundant expectoration of greenish or yellowish muco-purulent sputa. Expansion was deficient at right apex, but the vocal fremitus was not altered. The heart-sounds were normal. Not to carry

"On the Difficulties and Fallacies attending Physical Diagnosis in Diseases of the Chest." Gay's Hosp. Reports, series ii., vol. iv., p. 12. 
the signs and symptoms further, they were, according to my colleagues, who ordered his admission, and those of my clerks, the signs and symptoms of phthisis. On the other hand, there was the man's history, and, in spite of continued loss of flesh, the look, if I may be allowed to think so, of a patient with chronic bronchitis. But I am bound to confess that my diagnosis is uncertain. I think if he came, as I do not consider he will come, at any rate yet, to the postmortem table, we should find dilated bronchi and general bronchitis ; but $I$ should not be surprised if my colleagues and my clerks were proved to be more right than I.

I do not like to occupy time by comparing with this other cases. The question, of course, arises, have we nothing whereon to make a certain diagnosis? I am very much afraid we have not. There are certain distinctions laid down by Dr. Walshe and others which do give some aid, but that is very little, so far as my experience goes. In the character of the sputa there is nothing, I think, though streaks of blood occasionally, in the absence of any mitral mischief, may support the fear of phthisis. "The more limited the bronchitis, especially if situated at the apex, the greater the probability of its being associated with phthisis; the more general the bronchitis, especially if it affect both lungs, the greater the hope of an exemption from phthisis. The more abrupt the transition from bronchial obstruction to natural or puerile respiratory murmur in the affected part of a lung, the greater the likelihood of phthisis" 2 These and the fact that the tendency of bronchitis is to enlarge, that of phthisis to contract the chest, are possibly our only safeguards. And this contraction or enlargement is, in the main, anteroposterior.

On this latter point I may lay before you two plans of chest area; one, the larger, taken from the patient I have spoken of: the other from a patient with undoubted phthisis. In the first (R. R.) the lateral through measurement is 123 inches, the antero-posterior $9 \frac{1}{2}$ inches. In the second (J. J.), the lateral $12 \frac{3}{14}$, antero-posterior $7 \frac{5}{5}$. Between the first measurements is the difference of $\frac{21}{2} \frac{1}{8}$ of an inch; between the second that of $2 \frac{1}{8}$ inches. Both are taken at the level of the nipple line. The lung area is decidedly larger in $R$. R., whose height is 5 feet 9 inches, and weight 9 st. $5 \mathrm{lb}$. The height of the phthisical patient (J.J.) is 5 feet 6 inches, and his weight 8 st. $11 \mathrm{~b}$. In these two points the two men are fairly comparable.

And now I may be with some reason asked why I spend so much time over a distinction already well-drawn in all the text-books between these two diseases? My answer is, first of all because I believe the treatment by climate, especially of the two classes of patient, to be by no means the same; that the sending of a given patient to a certain high region, for example, will depend for the chance of a bettered condition on almost certain diagnosis between the two classes; and, secondly, that the difference and the distinction are often overlooked by some of the most practised among us, as I think the cases I have already quoted from various writers sufficiently prove. And as the result of an inaccurate discrimination, the relief of a bronchitis has led I am sure in no small number of cases to the hasty decision of really phthisical patients, in the absence or in spite of the advice of their medical attendant, to try that same mode of treatment, to their own detriment in the end. As proof of this it is justifiable to take the evidence, not of the medical, but of the general press. In the Morning Post of Dec. $26 \mathrm{th}, 1878$, is a by no means feeble or unfair article entitled "A Colony of Consumptives." It covers almost five columns of the newspaper. From THE LANCET I gather that something of the same kind has been published in the Echo. The correspondent of the Morning Post tells us that in this colony, "on any day half a dozen people of both sexes may be seen skating, who came here a month or two ago with extensive cavities in their lungs; cavities which, according to the local doctors, are fast disappearing" (col. 3.) Lower down the writer says, " the worst of Davos is that the people are always talking of 'cavities' and 'infiltration,' and "consolidation." "The loose employment of words which science itself scarcely as yet accurately defines may be but one of the signs of the times, and so far to be defended on newspaper gronnds, but it behoves us to decide with ourselves how far this popular knowledge, mischievous certainly in its immediate results, is not the outgrowth of our own want of precision, whether in diagnosis or in terminology.

In an article entitled "A Christmas at Davos Platz," Dr. Theodore Williams tells us that, among other symptoms of improvement in the patients he saw there, "the physical signs in both first and second stage cases of phthisis showed a remarkable diminution of dulness, indicating either the development of emphysema or absorption of pneumonic growth. Contraction of cavities appears to proceed somewhat rapidly here. But the climate seems to tell most on the heart's action, which becomes more and more excited, though, curious to state, the number of cases of hæmoptysis, even in phthisical patients, is very small." This opinion may carry some weight with " the public, who grasp eagerly at any novel idea which holds out any prospect of a cure for this disease." 4

I may agree to some extent with the writer as to the development of compensatory emphysema, or even as to the absorption of pneumonic products in cases of chronic phthisis. The latter, when it happily occurs, is hardly capable of post-mortem proof ; the former, so to say purely mechanical, is certainly a partial, and more or less temporary, relief to the patient. But when it does occur, it again brings the sufferer under the conditions of the persons called in the common language "asthmatic"-under the condition, that is to say, of the man or woman affected with chronic bronchitis and emphysema; and so far, I believe, under the conditions of the patient who has most to gain from the air and surroundings of a place like Davos, that gain being greatest to the marked chronic bronchitic, or to the certainly phthisical patient in whom the existing signs are rather those of bronchitis and emphysema than those of vesicular mischief, whatever be its so-called stage.

To conclude, and at the same time to revert to the first subject of this paper, there is a story told in Sir Astley Cooper's "Life" which ought, I think, to have some interest for, and, so far as I know, may have some bearing on the history of, the Society before which I have the honour to speak. It seems that Sir Astley Cooper ${ }^{5}$ was for a long period attached to the Kent Medical Society, which at that time held its meetings at the "Green Man" Hotel at Blackheath. Prevented by increase of professional work from attending, he sent in his resignation, addressed to it by some mistake as the Blackheath Medical Club. Dr. Babington and Professor Coleman proposed, and I suppose the Society unanimously adopted their proposal, that the resignation should not be accepted, on the ground that some other Society than their own must have been intended. The consequence of this very practical joke was that, remaining on the books up to Sept. 1829, he had to pay $£ 43$ as arrears of subscription. But it speaks well for the Society, in which he was thus proved to be so popular, that this fine did not prevent him from continuing to be an honorary member of it up to the time of his death.

\section{RINGWORM (OF THE HEAD) : DIAGNOSIS AND TREATMENT.}

By ALDER SMITH, M.B. LoNd., F.R.C.S., RESIDENT MEDICAX OFFICER AT CHRIST'S HOSPITAL, LONDON

HAVING devoted much attention, during the last nine years, to the diagnosis and treatment of ringworm, especially of chronic and inveterate cases, and being constantly asked by medical men as to the best treatment, I trust I may be able to give some practical hints on the subject; especially as to the production of kerion, the inflammatory form of the disease, which, if it can be produced artificially, always ends in a speedy cure.

DIAGNOSIS.

I do not propose to give a general description of the disease, as this may be obtained from many admirable books on the subject. But I cannot help remarking, in the first place, that very few medical men, either in consultation or private practice, are aware how incurable some cases of ringworm are; and the majority consider a case well when it is in a decidedly clironic state. Many boys are presented for admission to Christ's Hospital who, while bringing cer- 